\title{
Does dark matter consist of baryons of new stable family quarks?
}

\author{
G. Bregar, N.S. Mankoč Borštnik \\ Department of Physics, FMF, University of Ljubljana, Jadranska 19, 1000 Ljubljana
}

(Dated: April 21, 2022)

\begin{abstract}
We investigate the possibility that the dark matter consists of clusters of the heavy family quarks and leptons with zero Yukawa couplings to the lower families. Such a family is predicted by the approach unifying spin and charges as the fifth family. We make a rough estimation of properties of baryons of this new family members, of their behaviour during the evolution of the universe and when scattering on the ordinary matter and study possible limitations on the family properties due to the cosmological and direct experimental evidences.
\end{abstract}




\section{INTRODUCTION}

Although the origin of the dark matter is unknown, its gravitational interaction with the known matter and other cosmological observations require from the candidate for the dark matter constituent that: i. The scattering amplitude of a cluster of constituents with the ordinary matter and among the dark matter clusters themselves must be small enough, so that no effect of such scattering has been observed, except possibly in the DAMA/NaI [1] and not (yet?) in the CDMS and other experiments [2]. ii. Its density distribution (obviously different from the ordinary matter density distribution) causes that all the stars within a galaxy rotate approximately with the same velocity (suggesting that the density is approximately spherically symmetrically distributed, descending with the second power of the distance from the center, it is extended also far out of the galaxy, manifesting the gravitational lensing by galaxy clusters). iii. The dark matter constituents must be stable in comparison with the age of our universe, having obviously for many orders of magnitude different time scale for forming (if at all) solid matter than the ordinary matter. iv. The dark matter constituents had to be formed during the evolution of our universe so that they contribute today the main part of the matter ((5-7) times as much as the ordinary matter).

There are several candidates for the massive dark matter constituents in the literature, like, for example, WIMPs (weakly interacting massive particles), the references can be found in [1, 3]. In this paper we discuss the possibility that the dark matter constituents are clusters of a stable (from the point of view of the age of the universe) family of quarks and leptons. Such a family is predicted by the approach unifying spin and charges [5, 6, 8], proposed by one of the authors of this paper: N.S.M.B. This approach is showing a new way beyond the standard model of the electroweak and colour interactions by answering the open questions of this model like: Where do the families originate?, Why do only the left handed quarks and leptons carry the weak charge, while the right handed ones do not? Why do particles carry the observed $S U(2), U(1)$ and $S U(3)$ charges? Where does the Higgs field originate from?, and others.

There are several attempts in the literature trying to understand the origin of families. All of them, however, in one or another way (for example through choices of appropriate groups) simply postulate that there are at least three families, as does the standard model of the electroweak and colour interactions. Proposing the (right) mechanism for generating 
families is to our understanding the most promising guide to physics beyond the standard model.

The approach unifying spin and charges is offering the mechanism for the appearance of families. It introduces the second kind [5, 6, 7, 10] of the Clifford algebra objects, which generates families as the equivalent representations to the Dirac spinor representation. The references [7, 10] show that there are two, only two, kinds of the Clifford algebra objects, one used by Dirac to describe the spin of fermions. The second kind forms the equivalent representations with respect to the Lorentz group for spinors [5] and the families do form the equivalent representations with respect to the Lorentz group. The approach, in which fermions carry two kinds of spins (no charges), predicts from the simple starting action more than the observed three families. It predicts two times four families with masses several orders of magnitude bellow the unification scale of the three observed charges.

Since due to the approach (after assuming a particular, but to our opinion trustable, way of a nonperturbative breaking of the starting symmetry) the fifth family decouples in the Yukawa couplings from the lower four families (whose the fourth family quark's mass is predicted to be at around $250 \mathrm{GeV}$ or above [5, 8]), the fifth family quarks and leptons are stable as required by the condition iii.. Since the masses of all the members of the fifth family lie, due to the approach, much above the known three and the predicted fourth family masses, the baryons made out of the fifth family form small enough clusters (as we shall see in section II so that their scattering amplitude among themselves and with the ordinary matter is small enough and also the number of clusters (as we shall see in section III) is low enough to fulfil the conditions i. and iii.. Our study of the behaviour of the fifth family quarks in the cosmological evolution (section III) shows that also the condition iv. is fulfilled, if the fifth family masses are large enough.

Let us add that there are several assessments about masses of a possible (non stable) fourth family of quarks and leptons, which follow from the analyses of the existing experimental data and the cosmological observations. Although most of physicists have doubts about the existence of any more than the three observed families, the analyses clearly show that neither the experimental electroweak data [4, 15], nor the cosmological observations [4] forbid the existence of more than three families, as long as the masses of the fourth family quarks are higher than a few hundred $\mathrm{GeV}$ and the masses of the fourth family leptons above one hundred $\mathrm{GeV}\left(\nu_{4}\right.$ could be above $\left.50 \mathrm{GeV}\right)$. We studied in the references [5, 8, 9] possi- 
ble (non perturbative) breaks of the symmetries of the simple starting Lagrangean which, by predicting the Yukawa couplings, leads at low energies first to twice four families with no Yukawa couplings between these two groups of families. One group obtains at the last break masses of several hundred $\mathrm{TeV}$ or higher, while the lower four families stay massless and mass protected [9]. For one choice of the next break [8] the fourth family members $\left(u_{4}, d_{4}, \nu_{4}, e_{4}\right)$ obtain the masses at $(224 \mathrm{GeV}(285 \mathrm{GeV}), 285 \mathrm{GeV}(224 \mathrm{GeV}), 84 \mathrm{GeV}, 170$ $\mathrm{GeV}$ ), respectively. For the other choice of the next break we could not determine the fourth family masses, but when assuming the values for these masses we predicted mixing matrices in dependence on the masses. All these studies were done on the tree level. We are studying now symmetries of the Yukawa couplings if we go beyond the tree level. Let us add that the last experimental data [16] from the HERA experiments require that there is no $d_{4}$ quark with the mass lower than $250 \mathrm{GeV}$.

Our stable fifth family baryons, which might form the dark matter, also do not contradict the so far observed experimental data - as it is the measured (first family) baryon number and its ratio to the photon energy density, as long as the fifth family quarks are heavy enough $(>1 \mathrm{TeV})$. (This would be true for any stable heavy family.) Namely, all the measurements, which connect the baryon and the photon energy density, relate to the moment(s) in the history of the universe, when baryons of the first family where formed $\left(k_{b} T\right.$ bellow the binding energy of the three first family quarks dressed into constituent mass of $m_{q_{1}} c^{2} \approx 300$ $\mathrm{MeV}$, that is bellow $10 \mathrm{MeV})$ and the electrons and nuclei formed atoms $\left(k_{b} T \approx 1 \mathrm{eV}\right)$. The chargeless (with respect to the colour and electromagnetic charges) clusters of the fifth family were formed long before (at $k_{b} T \approx E_{c_{5}}$ (see Table $\left.\mathrm{I}\right)$ ), contributing the equal amount of the fifth family baryons and anti-baryons to the dark matter, provided that there is no fifth family baryon-anti-baryon asymmetry (if the asymmetry is nonzero the colourless baryons or anti-baryons are formed also at the early stage of the colour phase transition at around $1 \mathrm{GeV}$ ). They manifest after decoupling from the plasma (with their small number density and small cross section) (almost) only their gravitational interaction.

In this paper we estimate the properties of the fifth family members $\left(u_{5}, d_{5}, \nu_{5}, e_{5}\right)$, as well as of the clusters of these members, in particular the fifth family neutrons, under the assumptions that:

I. Neutron is the lightest fifth family baryon.

II. There is no fifth family baryon-anti-baryon asymmetry. 
The assumptions are made since we are not yet able to derive the properties of the family from the starting Lagrange density of the approach. The results of the present paper's study are helpful to better understand steps needed to come from the approach's starting Lagrange density to the low energy effective one.

From the approach unifying spin and charges we learn:

i. The stable fifth family members have masses higher than $\approx 1 \mathrm{TeV}$ and smaller than $\approx 10^{6}$ $\mathrm{TeV}$.

ii. The stable fifth family members have the properties of the lower four families; that is the same family members with the same (electromagnetic, weak and colour) charges and interacting correspondingly with the same gauge fields.

We estimate the masses of the fifth family quarks by studying their behaviour in the evolution of the universe, their formation of chargeless (with respect to the electromagnetic and colour interaction) clusters and the properties of these clusters when scattering on the ordinary (made mostly of the first family members) matter and among themselves. We use a simple (the hydrogen-like) model [11] to estimate the size and the binding energy of the fifth family baryons, assuming that the fifth family quarks are heavy enough to interact mostly by exchanging one gluon. We solve the Boltzmann equations for the fifth family quarks (and anti-quarks) forming the colourless clusters in the expanding universe, starting in the energy region when the fifth family members are ultrarelativistic, up to $\approx 1 \mathrm{GeV}$ when the colour phase transition starts. In this energy interval the one gluon exchange is the dominant interaction among quarks and the plasma. We conclude that the quarks and anti-quarks, which succeed to form neutral (colourless and electromagnetic chargeless) clusters, have the properties of the dark matter constituents if their masses are within the interval of a few $\mathrm{TeV}<m_{q_{5}} c^{2}<$ a few hundred $\mathrm{TeV}$, while the rest of the coloured fifth family objects annihilate within the colour phase transition period with their anti-particles for the zero fifth family baryon number asymmetry.

We estimate also the behaviour of our fifth family clusters if hitting the DAMA/NaIDAMA-LIBRA [1] and CDMS [2] experiments presenting the limitations the DAMA/NaI experiments put on our fifth family quarks when recognizing that CDMS has not found any event (yet).

The fifth family baryons are not the objects (WIMPS), which would interact with only the weak interaction, since their decoupling from the rest of the plasma in the expanding 
universe is determined by the colour force and their interaction with the ordinary matter is determined with the fifth family "nuclear force" (this is the force among clusters of the fifth family quarks, manifesting much smaller cross section than does the ordinary, mostly first family, "nuclear force") as long as their mass is not higher than $10^{4} \mathrm{TeV}$, when the weak interaction starts to dominate as commented in the last paragraph of section IV.

\section{PROPERTIES OF CLUSTERS OF THE HEAVY FAMILY}

Let us study the properties of the fifth family of quarks and leptons as predicted by the approach unifying spin and charges, with masses several orders of magnitude greater than those of the known three families, decoupled in the Yukawa couplings from the lower mass families and with the charges and their couplings to the gauge fields of the known families (which all seems, due to our estimate predictions of the approach, reasonable assumptions). Families distinguish among themselves (besides in masses) in the family index (in the quantum number, which in the approach is determined by the second kind of the Clifford algebra objects' operators [5, 6, 7] $\tilde{S}^{a b}=\frac{i}{4}\left(\tilde{\gamma}^{a} \tilde{\gamma}^{b}-\tilde{\gamma}^{b} \tilde{\gamma}^{a}\right)$, anti-commuting with the Dirac $\gamma^{a}$ 's), and (due to the Yukawa couplings) in their masses.

For a heavy enough family the properties of baryons (protons $p_{5}\left(u_{5} u_{5} d_{5}\right)$, neutrons $n_{5}$ $\left.\left(u_{5} d_{5} d_{5}\right), \Delta_{5}^{-}, \Delta_{5}^{++}\right)$made out of quarks $u_{5}$ and $d_{5}$ can be estimated by using the non relativistic Bohr-like model with the $\frac{1}{r}$ dependence of the potential between a pair of quarks $V=-\frac{2}{3} \frac{\hbar c \alpha_{c}}{r}$, where $\alpha_{c}$ is in this case the colour coupling constant. Equivalently goes for anti-quarks. This is a meaningful approximation as long as the one gluon exchange is the dominant contribution to the interaction among quarks, that is as long as excitations of a cluster are not influenced by the linearly rising part of the potential [22]. The electromagnetic and weak interaction contributions are of the order of $10^{-2}$ times smaller. Which one of $p_{5}$, $n_{5}$, or maybe $\Delta_{5}^{-}$or $\Delta_{5}^{++}$, is a stable fifth family baryon, depends on the ratio of the bare masses $m_{u_{5}}$ and $m_{d_{5}}$, as well as on the weak and the electromagnetic interactions among quarks. If $m_{d_{5}}$ is appropriately smaller than $m_{u_{5}}$ so that the weak and electromagnetic interactions favor the neutron $n_{5}$, then $n_{5}$ is a colour singlet electromagnetic chargeless stable cluster of quarks, with the weak charge $-1 / 2$. If $m_{d_{5}}$ is larger (enough, due to the stronger electromagnetic repulsion among the two $u_{5}$ than among the two $d_{5}$ ) than $m_{u_{5}}$, the proton $p_{5}$ which is a colour singlet stable nucleon with the weak charge $1 / 2$, needs the 
electron $e_{5}$ or $e_{1}$ or $\bar{p}_{1}$ to form a stable electromagnetic chargeless cluster (in the last case it could also be the weak singlet and would accordingly manifest the ordinary nuclear force only). An atom made out of only fifth family members might be lighter or not than $n_{5}$, depending on the masses of the fifth family members.

Neutral (with respect to the electromagnetic and colour charge) fifth family particles that constitute the dark matter can be $n_{5}, \nu_{5}$ or charged baryons like $p_{5}, \Delta_{5}^{++}, \Delta_{5}^{-}$, forming neutral atoms with $e_{5}^{-}$or $\bar{e}_{5}^{+}$, correspondingly, or (as said above) $p_{5} \bar{p}_{1}$. We treat the case that $n_{5}$ as well as $\bar{n}_{5}$ form the major part of the dark matter, assuming that $n_{5}$ (and $\left.\bar{n}_{5}\right)$ are stable baryons (anti-baryons). Taking $m_{\nu_{5}}<m_{e_{5}}$ also $\nu_{5}$ contributes to the dark matter. We shall comment this in section $\mathrm{V}$.

In the Bohr-like model we obtain if neglecting more than one gluon exchange contribution

$$
E_{c 5} \approx-3 \frac{1}{2}\left(\frac{2}{3} \alpha_{c}\right)^{2} \frac{m_{q_{5}}}{2} c^{2}, \quad r_{c 5} \approx \frac{\hbar c}{\frac{2}{3} \alpha_{c} \frac{m_{q_{5}}}{2} c^{2}}
$$

The mass of the cluster is approximately $m_{c_{5}} c^{2} \approx 3 m_{q_{5}} c^{2}\left(1-\left(\frac{1}{3} \alpha_{c}\right)^{2}\right)$. We use the factor of $\frac{2}{3}$ for a two quark pair potential and of $\frac{4}{3}$ for a quark and an anti-quark pair potential. If treating correctly the three quarks' (or anti-quarks') center of mass motion in the hydrogen-like model, allowing the hydrogen-like functions to adapt the width as presented in Appendix I, the factor $-3 \frac{1}{2}\left(\frac{2}{3}\right)^{2} \frac{1}{2}$ in Eq. 1 is replaced by 0.66, and the mass of the cluster is accordingly $3 m_{q_{5}} c^{2}\left(1-0.22 \alpha_{c}^{2}\right)$, while the average radius takes the values as presented in Table I.

Assuming that the coupling constant of the colour charge $\alpha_{c}$ runs with the kinetic energy $-E_{c_{5}} / 3$ and taking into account the number of families which contribute to the running coupling constant in dependence on the kinetic energy (and correspondingly on the mass of the fifth family quarks) we estimate the properties of a baryon as presented on Table I (the table is calculated from the hydrogen-like model presented in Appendix I),

The binding energy is approximately $\frac{1}{100}$ of the mass of the cluster (it is $\approx \frac{\alpha_{c}^{2}}{3}$ ). The baryon $n_{5}\left(u_{5} d_{5} d_{5}\right)$ is lighter than the baryon $p_{5},\left(u_{q_{5}} d_{q_{5}} d_{q_{5}}\right)$ if $\Delta m_{u d}=\left(m_{u_{5}}-m_{d_{5}}\right)$ is smaller than $\approx(0.05,0.5,5,50,500,5000) \mathrm{GeV}$ for the six values of the $m_{q_{5}} c^{2}$ on Table $\mathrm{I}$, respectively. We see from Table [ that the "nucleon-nucleon" force among the fifth family baryons leads to many orders of magnitude smaller cross section than in the case of the first family nucleons $\left(\sigma_{c_{5}}=\pi r_{c 5}^{2}\right.$ is from $10^{-5} \mathrm{fm}^{2}$ for $m_{q_{5}} c^{2}=1 \mathrm{TeV}$ to $10^{-14} \mathrm{fm}^{2}$ for $m_{q_{5}} c^{2}=10^{5}$ $\mathrm{TeV}$ ). Accordingly is the scattering cross section between two fifth family baryons determined by the weak interaction as soon as the mass exceeds several $\mathrm{GeV}$. 


\begin{tabular}{|c||c|c|c|c|}
\hline$\frac{m_{q_{5}} c^{2}}{\mathrm{TeV}}$ & $\alpha_{c}$ & $\frac{E_{c_{5}}}{m_{q_{5}} c^{2}}$ & $\frac{r_{c_{5}}}{10^{-6} \mathrm{fm}}$ & $\frac{\Delta m_{u d} c^{2}}{\mathrm{GeV}}$ \\
\hline \hline 1 & 0.16 & -0.016 & $3.2 \cdot 10^{3}$ & 0.05 \\
\hline 10 & 0.12 & -0.009 & $4.2 \cdot 10^{2}$ & 0.5 \\
\hline $10^{2}$ & 0.10 & -0.006 & 52 & 5 \\
\hline $10^{3}$ & 0.08 & -0.004 & 6.0 & 50 \\
\hline $10^{4}$ & 0.07 & -0.003 & 0.7 & $5 \cdot 10^{2}$ \\
\hline $10^{5}$ & 0.06 & -0.003 & 0.08 & $5 \cdot 10^{3}$ \\
\hline
\end{tabular}

TABLE I: The properties of a cluster of the fifth family quarks within the extended Bohr-like (hydrogen-like) model from Appendix I. $m_{q_{5}}$ in $\mathrm{TeV} / \mathrm{c}^{2}$ is the assumed fifth family quark mass, $\alpha_{c}$ is the coupling constant of the colour interaction at $E \approx\left(-E_{c_{5}} / 3\right)$ (Eq1) which is the kinetic energy of quarks in the baryon, $r_{c_{5}}$ is the corresponding average radius. Then $\sigma_{c_{5}}=\pi r_{c_{5}}^{2}$ is the corresponding scattering cross section.

If a cluster of the heavy (fifth family) quarks and leptons and of the ordinary (the lightest) family is made, then, since ordinary family dictates the radius and the excitation energies of a cluster, its properties are not far from the properties of the ordinary hadrons and atoms, except that such a cluster has the mass dictated by the heavy family members.

\section{EVOLUTION OF THE ABUNDANCE OF THE FIFTH FAMILY MEMBERS IN THE UNIVERSE}

We assume that there is no fifth family baryon-anti-baryon asymmetry and that the neutron is the lightest baryon made out of the fifth family quarks. Under these assumptions and with the knowledge from our rough estimations [8 that the fifth family masses are within the interval from $1 \mathrm{TeV}$ to $10^{6} \mathrm{TeV}$ we study the behaviour of our fifth family quarks and anti-quarks in the expanding (and accordingly cooling down [3]) universe in the plasma of all other fields (fermionic and bosonic) from the period, when the fifth family members carrying all the three charges (the colour, weak and electromagnetic) are ultra relativistic and is their number (as there are the numbers of all the other fermions and bosons in the ultra relativistic regime) determined by the temperature. We follow the fifth family quarks and anti-quarks first through the freezing out period, when the fifth family quarks and anti- 
quarks start to have too large mass to be formed out of the plasma (due to the plasma's too low temperature), then through the period when first the clusters of di-quarks and di-antiquarks and then the colourless neutrons and anti-neutrons $\left(n_{5}\right.$ and $\left.\bar{n}_{5}\right)$ are formed. The fifth family neutrons being tightly bound into the colourless objects do not feel the colour phase transition when it starts bellow $k_{b} T \approx 1 \mathrm{GeV}\left(k_{b}\right.$ is the Boltzmann constant) and decouple accordingly from the rest of quarks and anti-quarks and gluons and manifest today as the dark matter constituents. We take the quark mass as a free parameter in the interval from $1 \mathrm{TeV}$ to $10^{6} \mathrm{TeV}$ and determine the mass from the observed dark matter density.

At the colour phase transition, however, the coloured fifth family quarks and anti-quarks annihilate to the today's unmeasurable density: Heaving much larger mass (of the order of $10^{5}$ times larger), and correspondingly much larger momentum (of the order of $10^{3}$ times larger) as well as much larger binding energy (of the order of $10^{5}$ times larger) than the first family quarks when they are "dressed" into constituent mass, the coloured fifth family quarks succeed in the colour phase transition region to annihilate with the corresponding anti-quarks to the non measurable extend, if it is no fifth family baryon asymmetry.

In the freezing out period almost up to the colour phase transition the kinetic energy of quarks is high enough so that the one gluon exchange dominates in the colour interaction of quarks with the plasma, while the (hundred times) weaker weak and electromagnetic interaction can be neglected.

The quarks and anti-quarks start to freeze out when the temperature of the plasma falls close to $m_{q_{5}} c^{2} / k_{b}$. They are forming clusters (bound states) when the temperature falls close to the binding energy (which is due to Table $\mathrm{I} \approx \frac{1}{100} m_{q_{5}} c^{2}$ ). When the three quarks (or three anti-quarks) of the fifth family form a colourless baryon (or anti-baryon), they decouple from the rest of plasma due to small scattering cross section manifested by the average radius presented in Table 1 .

Recognizing that at the temperatures $\left(10^{6} \mathrm{TeV}>k_{b} T>1 \mathrm{GeV}\right)$ the one gluon exchange gives the dominant contribution to the interaction among quarks of any family, it is not difficult to estimate the thermally averaged scattering cross sections (as the function of the temperature) for the fifth family quarks and anti-quarks to scatter:

i. into all the relativistic quarks and anti-quarks of lower mass families $\left.(<\sigma v\rangle_{q \bar{q}}\right)$, ii. into gluons $\left.(<\sigma v\rangle_{g g}\right)$,

iii. into (annihilating) bound states of a fifth family quark and an anti-quark mesons 
$\left(<\sigma v>_{(q \bar{q})_{b}}\right)$,

iv. into bound states of two fifth family quarks and into the fifth family baryons $\left.(<\sigma v\rangle_{c_{5}}\right)$ (and equivalently into two anti-quarks and into anti-baryons).

The one gluon exchange scattering cross sections are namely (up to the strength of the coupling constants and up to the numbers of the order one determined by the corresponding groups) equivalent to the corresponding cross sections for the one photon exchange scattering cross sections, and we use correspondingly also the expression for scattering of an electron and a proton into the bound state of a hydrogen when treating the scattering of two quarks into the bound states. We take the roughness of such estimations into account by two parameters: The parameter $\eta_{c_{5}}$ takes care of scattering of two quarks (anti-quarks) into three colourless quarks (or anti-quarks), which are the fifth family baryons (anti-baryons) and about the uncertainty with which this cross section is estimated. $\eta_{(q \bar{q})_{b}}$ takes care of the roughness of the used formula for $\langle\sigma v\rangle_{(q \bar{q})_{b}}$.

The following expressions for the thermally averaged cross sections are used

$$
\begin{aligned}
<\sigma v>_{q \bar{q}} & =\frac{16 \pi}{9}\left(\frac{\alpha_{c} \hbar c}{m_{q_{5}} c^{2}}\right)^{2} c \\
<\sigma v>_{g g} & =\frac{37 \pi}{108}\left(\frac{\alpha_{c} \hbar c}{m_{q_{5}} c^{2}}\right)^{2} c \\
<\sigma v>_{c_{5}} & =\eta_{c_{5}} 10\left(\frac{\alpha_{c} \hbar c}{m_{q_{5}} c^{2}}\right)^{2} c \sqrt{\frac{E_{c_{5}}}{k_{b} T}} \ln \frac{E_{c_{5}}}{k_{b} T}, \\
<\sigma v>_{(q \bar{q})_{b}} & =\eta_{(q \bar{q})_{b}} 10\left(\frac{\alpha_{c} \hbar c}{m_{q_{5}} c^{2}}\right)^{2} c \sqrt{\frac{E_{c_{5}}}{k_{b} T}} \ln \frac{E_{c_{5}}}{k_{b} T}, \\
\sigma_{T} & =\frac{8 \pi}{3}\left(\frac{\alpha_{c} \hbar c}{m_{q_{5}} c^{2}}\right)^{2},
\end{aligned}
$$

where $v$ is the relative velocity between the fifth family quark and its anti-quark, or between two quarks and $E_{c_{5}}$ is the binding energy for a cluster (Eq. 1). $\sigma_{T}$ is the Thompson-like scattering cross section of gluons on quarks (or anti-quarks).

To see how many fifth family quarks and anti-quarks of a chosen mass form the fifth family baryons and anti-baryons today we solve the coupled systems of Boltzmann equations presented bellow as a function of time (or temperature). The value of the fifth family quark mass which predicts the today observed dark matter is the mass we are looking for. Due to the inaccuracy of the estimated scattering cross sections entering into the Boltzmann equations we tell the interval within which the mass lies. We follow in our derivation of the 
Boltzmann equations (as much as possible) the ref. [3], chapter 3.

Let $T_{0}$ be the today's black body radiation temperature, $T(t)$ the actual (studied) temperature, $a^{2}\left(T^{0}\right)=1$ and $a^{2}(T)=a^{2}(T(t))$ is the metric tensor component in the expanding flat universe - the Friedmann-Robertson-Walker metric: $\operatorname{diag} g_{\mu \nu}=\left(1,-a(t)^{2},-a(t)^{2},-a(t)^{2}\right)$, $\left(\frac{\dot{a}}{a}\right)^{2}=\frac{8 \pi G}{3} \rho$, with $\rho=\frac{\pi^{2}}{15} g^{*} T^{4}, T=T(t), g^{*}$ measures the number of degrees of freedom of those of the four family members (f) and gauge bosons (b), which are at the treated temperature $T$ ultra-relativistic $\left(g^{*}=\sum_{i \in \mathrm{b}} g_{i}+\frac{7}{8} \sum_{i \in \mathrm{f}} g_{i}\right) . H_{0} \approx 1.5 \cdot 10^{-42} \frac{\mathrm{GeV} c}{\hbar c}$ is the present Hubble constant and $G=\frac{\hbar c}{\left(m_{p l}^{2}\right)}, m_{p l} c^{2}=1.2 \cdot 10^{19} \mathrm{GeV}$.

Let us write down the Boltzmann equation, which treats in the expanding universe the number density of all the fifth family quarks as a function of time $t$. The fifth family quarks scatter with anti-quarks into all the other relativistic quarks (with the number density $n_{q}$ ) and anti-quarks $\left(n_{\bar{q}}\left(\langle\sigma v\rangle_{q \bar{q}}\right)\right.$ and into gluons $\left.(<\sigma v\rangle_{g g}\right)$. At the beginning, when the quarks are becoming non-relativistic and start to freeze out, the formation of bound states is negligible. One finds [3] the Boltzmann equation for the fifth family quarks $n_{q_{5}}$ (and equivalently for anti-quarks $n_{\bar{q}_{5}}$ )

$$
\begin{aligned}
a^{-3} \frac{d\left(a^{3} n_{q_{5}}\right)}{d t}= & <\sigma v>_{q \bar{q}} n_{q_{5}}^{(0)} n_{\bar{q}_{5}}^{(0)}\left(-\frac{n_{q_{5}} n_{\bar{q}_{5}}}{n_{q_{5}}^{(0)} n_{\bar{q}_{5}}^{(0)}}+\frac{n_{q} n_{\bar{q}}}{n_{q}^{(0)} n_{\bar{q}}^{(0)}}\right)+ \\
& <\sigma v>_{g g} n_{q_{5}}^{(0)} n_{\bar{q}_{5}}^{(0)}\left(-\frac{n_{q_{5}} n_{\bar{q}_{5}}}{n_{q_{5}}^{(0)} n_{\bar{q}_{5}}^{(0)}}+\frac{n_{g} n_{g}}{n_{g}^{(0)} n_{g}^{(0)}}\right) .
\end{aligned}
$$

Let us tell that $n_{i}^{(0)}=g_{i}\left(\frac{m_{i} c^{2} k_{b} T}{(\hbar c)^{2}}\right)^{\frac{3}{2}} e^{-\frac{m_{i} c^{2}}{k_{b} T}}$ for $m_{i} c^{2}>>k_{b} T$ and $\frac{g_{i}}{\pi^{2}}\left(\frac{k_{b} T}{\hbar c}\right)^{3}$ for $m_{i} c^{2}<<$ $k_{b} T$. Since the ultra-relativistic quarks and anti-quarks of the lower families are in the thermal equilibrium with the plasma and so are gluons, it follows $\frac{n_{q} n_{\bar{q}}}{n_{q}^{(0)} n_{\bar{q}}^{(0)}}=1=\frac{n_{g} n_{g}}{n_{g}^{(0)} n_{g}^{(0)}}$. Taking into account that $(a T)^{3} g^{*}(T)$ is a constant it is appropriate [3] to introduce a new parameter $x=\frac{m_{q_{5}} c^{2}}{k_{b} T}$ and the quantity $Y_{q_{5}}=n_{q_{5}}\left(\frac{\hbar c}{k_{b} T}\right)^{3}, Y_{q_{5}}^{(0)}=n_{q_{5}}^{(0)}\left(\frac{\hbar c}{k_{b} T}\right)^{3}$. When taking into account that the number of quarks is the same as the number of anti-quarks, and that $\frac{d x}{d t}=\frac{h_{m} m_{q_{5}} c^{2}}{x}$, with $h_{m}=\sqrt{\frac{4 \pi^{3} g^{*}}{45}} \frac{c}{\hbar c m_{p l} c^{2}}$, Eq. 3 transforms into $\frac{d Y_{q_{5}}}{d x}=\frac{\lambda_{q_{5}}}{x^{2}}\left(Y_{q_{5}}^{(0) 2}-Y_{q_{5}}^{2}\right)$, with $\lambda_{q_{5}}=\frac{\left.\left(\langle\sigma v\rangle_{q \bar{q}}+<\sigma v\right\rangle_{g g}\right) m_{q_{5}} c^{2}}{h_{m}(\hbar c)^{3}}$. It is this equation which we are solving (up to the region of $x$ when the clusters of quarks and anti-quarks start to be formed) to see the behaviour of the fifth family quarks as a function of the temperature.

When the temperature of the expanding universe falls close enough to the binding energy of the cluster of the fifth family quarks (and anti-quarks), the bound states of quarks (and 
anti-quarks) and the clusters of fifth family baryons (in our case neutrons $n_{5}$ ) (and antibaryons $\bar{n}_{5}$-anti-neutrons) start to form. To a fifth family di-quark $\left(q_{5}+q_{5} \rightarrow\right.$ di-quark + gluon) a third quark clusters ( di-quark $+q_{5} \rightarrow c_{5}+$ gluon) to form the colourless fifth family neutron (anti-neutron), in an excited state (contributing gluons back into the plasma in the thermal bath when going into the ground state), all in thermal equilibrium. Similarly goes with the anti-quarks clusters. We take into account both processes approximately within the same equation of motion by correcting the averaged amplitude $\langle\sigma v\rangle_{c_{5}}$ for quarks to scatter into a bound state of di-quarks with the parameter $\eta_{c_{5}}$, as explained above. The corresponding Boltzmann equation for the number of baryons $n_{c_{5}}$ then reads

$$
a^{-3} \frac{d\left(a^{3} n_{c_{5}}\right)}{d t}=<\sigma v>_{c_{5}} n_{q_{5}}^{(0)^{2}}\left(\left(\frac{n_{q_{5}}}{n_{q_{5}}^{(0)}}\right)^{2}-\frac{n_{c_{5}}}{n_{c_{5}}^{(0)}}\right) .
$$

Introducing again $Y_{c_{5}}=n_{c_{5}}\left(\frac{\hbar c}{k_{b} T}\right)^{3}, Y_{c 5}^{(0)}=n_{c_{5}}^{(0)}\left(\frac{\hbar c}{k_{b} T}\right)^{3}$ and $\lambda_{c_{5}}=\frac{\langle\sigma v\rangle_{c_{5}} m_{q_{5}} c^{2}}{h_{m}(\hbar c)^{3}}$, with the same $x$ and $h_{m}$ as above, we obtain the equation $\frac{d Y_{c_{5}}}{d x}=\frac{\lambda_{c_{5}}}{x^{2}}\left(Y_{q_{5}}^{2}-Y_{c_{5}} Y_{q_{5}}^{(0)} \frac{Y_{q_{5}}^{(0)}}{Y_{c_{5}}^{(0)}}\right)$.

The number density of the fifth family quarks $n_{q_{5}}$ (and correspondingly $Y_{q_{5}}$ ), which has above the temperature of the binding energy of the clusters of the fifth family quarks (almost) reached the decoupled value, starts to decrease again due to the formation of the clusters of the fifth family quarks (and anti-quarks) as well as due to forming the bound state of the fifth family quark with an anti-quark, which annihilates into gluons. It follows

$$
\begin{aligned}
a^{-3} \frac{d\left(a^{3} n_{q_{5}}\right)}{d t}= & <\sigma v>_{c_{5}} n_{q_{5}}^{(0)} n_{q_{5}}^{(0)}\left[-\left(\frac{n_{q_{5}}}{n_{q_{5}}^{(0)}}\right)^{2}+\frac{n_{c_{5}}}{n_{c_{5}}^{(0)}}-\frac{\eta_{(q \bar{q})_{b}}}{\eta_{c_{5}}}\left(\frac{n_{q_{5}}}{n_{q_{5}}^{(0)}}\right)^{2}\right]+ \\
& <\sigma v>_{q \bar{q}} n_{q_{5}}^{(0)} n_{\bar{q}_{5}}^{(0)}\left(-\frac{n_{q_{5}} n_{\bar{q}_{5}}}{n_{q_{5}}^{(0)} n_{\bar{q}_{5}}^{(0)}}+\frac{n_{q} n_{\bar{q}}}{n_{q}^{(0)} n_{\bar{q}}^{(0)}}\right)+ \\
& <\sigma v>_{g g} n_{q_{5}}^{(0)} n_{\overline{q_{5}}}^{(0)}\left(-\frac{n_{q_{5}} n_{\bar{q}_{5}}}{n_{q_{5}}^{(0)} n_{\bar{q}_{5}}^{(0)}}+\frac{n_{g} n_{g}}{n_{g}^{(0)} n_{g}^{(0)}}\right)
\end{aligned}
$$

with $\eta_{(q \bar{q})_{b}}$ and $\eta_{c_{5}}$ defined in Eq. 2, Introducing the above defined $Y_{q_{5}}$ and $Y_{c_{5}}$ the Eq. 5 transforms into $\frac{d Y_{q_{5}}}{d x}=\frac{\lambda_{c_{5}}}{x^{2}}\left(-Y_{q_{5}}^{2}+Y_{c_{5}} Y_{q_{5}}^{(0)} \frac{Y_{q_{5}}^{(0)}}{Y_{c_{5}}^{(0)}}\right)+\frac{\lambda_{(q \bar{q})_{b}}}{x^{2}}\left(-Y_{q_{5}}^{2}\right)+\frac{\lambda_{q_{5}}}{x^{2}}\left(Y_{q_{5}}^{(0) 2}-Y_{q_{5}}^{2}\right)$, with $\lambda_{(q \bar{q})_{b}}=$ $\frac{\langle\sigma v\rangle_{(q \bar{q})_{b}} m_{q_{5}} c^{2}}{h_{m}(\hbar c)^{3}}$ (and with the same $x$ and $h_{m}$ as well as $\lambda_{c_{5}}$ and $\lambda_{q_{5}}$ as defined above). We solve this equation together with the above equation for $Y_{c_{5}}$.

Solving the Boltzmann equations (Eqs. 3, 4, 5) we obtain the number density of the fifth family quarks $n_{q_{5}}$ (and anti-quarks) and the number density of the fifth family baryons $n_{c_{5}}$ (and anti-baryons) as a function of the parameter $x=\frac{m_{q_{5}} c^{2}}{k_{b} T}$ and the two parameters $\eta_{c_{5}}$ and 
$\eta_{(q \bar{q})_{b}}$. The evaluations are made, as we explained above, with the approximate expressions for the thermally averaged cross sections from Eq.(2), corrected by the parameters $\eta_{c_{5}}$ and $\eta_{(q \bar{q})_{b}}$ (Eq. 2). We made a rough estimation of the two intervals, within which the parameters $\eta_{c_{5}}$ and $\eta_{(q \bar{q})_{b}}$ (Eq. 2) seem to be acceptable. More accurate evaluations of the cross sections are under consideration. In fig. III both number densities (multiplied by $\left(\frac{\hbar c}{k_{b} T}\right)^{3}$, which is $Y_{q_{5}}$ and $Y_{c_{5}}$, respectively for the quarks and the clusters of quarks) as a function of $\frac{m_{q_{5}} c^{2}}{k_{b} T}$ for $\eta_{(q \bar{q})_{3}}=1$ and $\eta_{c_{5}}=\frac{1}{50}$ are presented. The particular choice of the parameters $\eta_{(q \bar{q})_{3}}$ and $\eta_{c_{5}}$ in fig. III is made as a typical example. The calculation is performed up to $k_{b} T=1$ $\mathrm{GeV}$ (when the colour phase transition starts and the one gluon exchange stops to be the acceptable approximation).

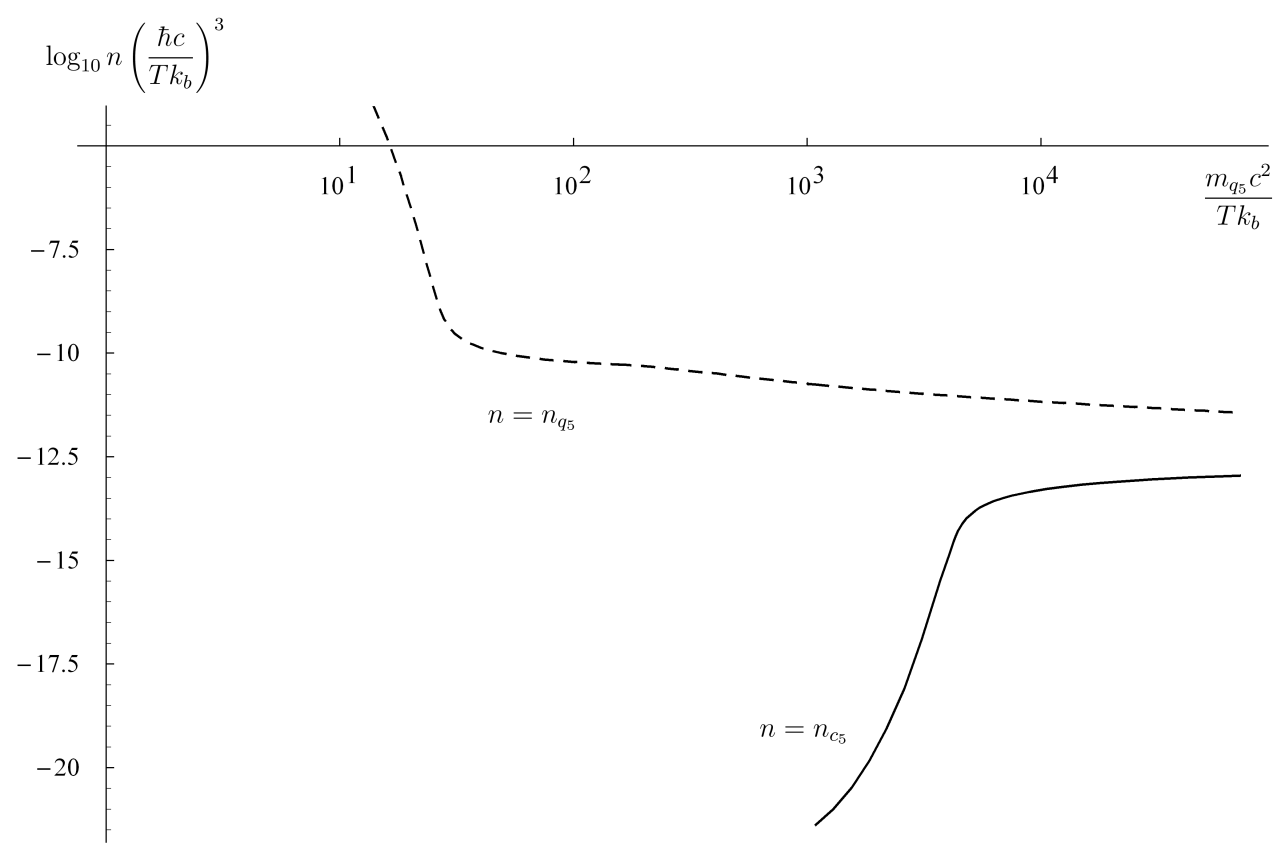

FIG. 1: The dependence of the two number densities $n_{q_{5}}$ (of the fifth family quarks) and $n_{c_{5}}$ (of the fifth family clusters) as function of $\frac{m_{q_{5}} c^{2}}{k_{b} T}$ is presented for the special values $m_{q_{5}} c^{2}=71 \mathrm{TeV}$, $\eta_{c_{5}}=\frac{1}{50}$ and $\eta_{(q \bar{q})_{b}}=1$. We take $g^{*}=91.5$. In the treated energy (temperature $k_{b} T$ ) interval the one gluon exchange gives the main contribution to the scattering cross sections of Eq.(2) entering into the Boltzmann equations for $n_{q_{5}}$ and $n_{c_{5}}$. In the figure we make a choice of the parameters within the estimated intervals.

Let us repeat how the $n_{5}$ and $\bar{n}_{5}$ evolve in the evolution of our universe. The quarks and 
anti-quarks are at high temperature $\left(\frac{m_{q_{5}} c^{2}}{k_{b} T}<<1\right)$ in thermal equilibrium with the plasma (as are also all the other families and bosons of lower masses). As the temperature of the plasma (due to the expansion of the universe) drops close to the mass of the fifth family quarks, quarks and anti-quarks scatter into all the other (ultra) relativistic fermions and bosons, but can not be created any longer from the plasma (in the average). At the temperature close to the binding energy of the quarks in a cluster, the clusters of the fifth family $\left(n_{c_{5}}, n_{\bar{c}_{5}}\right)$ baryons start to be formed. We evaluated the number density $n_{q_{5}}(T)\left(\frac{\hbar c}{k_{b} T}\right)^{3}=Y_{q_{5}}$ of the fifth family quarks (and anti-quarks) and the number density of the fifth family baryons $n_{c_{5}}(T)\left(\frac{\hbar c}{k_{b} T}\right)^{3}=Y_{c_{5}}$ for several choices of $m_{q_{5}}, \eta_{c_{5}}$ and $\eta_{(q \bar{q})_{b}}$ up to $k_{b} T_{l i m}=1 \mathrm{GeV}=\frac{m_{q_{5}} c^{2}}{x_{l i m}}$.

From the calculated decoupled number density of baryons and anti-baryons of the fifth family quarks (and anti-quarks) $n_{c_{5}}\left(T_{1}\right)$ at temperature $k_{b} T_{1}=1 \mathrm{GeV}$, where we stopped our calculations as a function of the quark mass and of the two parameters $\eta_{c_{5}}$ and $\eta_{(q \bar{q})_{b}}$, the today's mass density of the dark matter follows (after taking into account that when once the $n_{5}$ and $\bar{n}_{5}$ decouple, their number stays unchanged but due to the expansion of the universe their density decreases according to $a_{1}^{3} n_{c_{5}}\left(T_{1}\right)=a_{2}^{3} n_{c_{5}}\left(T_{2}\right)$, with the today's $a_{0}=1$ and the temperature $T_{0}=2.725^{\circ} \mathrm{K}$ ) leading to [3]

$$
\rho_{d m}=\Omega_{d m} \rho_{c r}=2 m_{c_{5}} n_{c_{5}}\left(T_{1}\right)\left(\frac{T_{0}}{T_{1}}\right)^{3} \frac{g^{*}\left(T_{1}\right)}{g^{*}\left(T_{0}\right)},
$$

where we take into account that $g^{*}\left(T_{1}\right)\left(a_{1} T_{1}\right)^{3}=g^{*}\left(T_{0}\right)\left(a_{0} T_{0}\right)^{3}$, with $T_{0}=2.5 \cdot 10^{-4} \frac{\mathrm{eV}}{k_{b}}$, $g^{*}\left(T_{0}\right)=2+\frac{7}{8} \cdot 3 \cdot\left(\frac{4}{11}\right)^{4 / 3}, g^{*}\left(T_{1}\right)=2+2 \cdot 8+\frac{7}{8}(5 \cdot 3 \cdot 2 \cdot 2+6 \cdot 2 \cdot 2)$ and $\rho_{c r} c^{2} \approx \frac{3 H_{0}^{2} c^{2}}{8 \pi G} \approx$ $5.7 \cdot 10^{3} \frac{\mathrm{eV}}{\mathrm{cm}^{3}}$, factor 2 counts baryons and anti-baryons.

The intervals for the acceptable parameters $\eta_{c_{5}}$ and $\eta_{(q \bar{q})_{b}}$ (determining the inaccuracy, with which the scattering cross sections were evaluated) influence the value of $n_{c_{5}}$ and determine the interval, within which one expects the fifth family mass. We read from Table II the mass interval for the fifth family quarks' mass, which fits Eqs. (6, 4, 3):

$$
10 \mathrm{TeV}<m_{q_{5}} c^{2}<\text { a few } \cdot 10^{2} \mathrm{TeV} .
$$

From this mass interval we estimate from Table [ the cross section for the fifth family neutrons $\pi\left(r_{c_{5}}\right)^{2}$ :

$$
10^{-8} \mathrm{fm}^{2}<\sigma_{c_{5}}<10^{-6} \mathrm{fm}^{2}
$$

(It is at least $10^{-6}$ smaller than the cross section for the first family neutrons.) 


\begin{tabular}{|c||c|c|c|c|c|}
\hline$\frac{m_{q_{5}} c^{2}}{\mathrm{TeV}}$ & $\eta_{(q \bar{q})_{b}}=\frac{1}{10}$ & $\eta_{(q \bar{q})_{b}}=\frac{1}{3}$ & $\eta_{(q \bar{q})_{b}}=1$ & $\eta_{(q \bar{q})_{b}}=3$ & $\eta_{(q \bar{q})_{b}}=10$ \\
\hline \hline$\eta_{c_{5}}=\frac{1}{50}$ & 21 & 36 & 71 & 159 & 417 \\
\hline$\eta_{c_{5}}=\frac{1}{10}$ & 12 & 20 & 39 & 84 & 215 \\
\hline$\eta_{c_{5}}=\frac{1}{3}$ & 9 & 14 & 25 & 54 & 134 \\
\hline$\eta_{c_{5}}=1$ & 8 & 11 & 19 & 37 & 88 \\
\hline$\eta_{c_{5}}=3$ & 7 & 10 & 15 & 27 & 60 \\
\hline$\eta_{c_{5}}=10$ & $7^{*}$ & $8^{*}$ & 13 & 22 & 43 \\
\hline
\end{tabular}

TABLE II: The fifth family quark mass is presented (Eq. (6) ), calculated for different choices of $\eta_{c_{5}}$ (which takes care of the inaccuracy with which a colourless cluster of three quarks (anti-quarks) cross section was estimated and of $\eta_{(q \bar{q})_{b}}$ (which takes care of the inaccuracy with which the cross section for the annihilation of a bound state of quark - anti-quark was taken into account) from Eqs. 6, 4, 3). * denotes non stable calculations.

Let us comment on the fifth family quark - anti-quark annihilation at the colour phase transition, which starts at approximately $1 \mathrm{GeV}$. When the colour phase transition starts, the quarks start to "dress" into constituent mass, which brings to them $\approx 300 \mathrm{MeV} / \mathrm{c}^{2}$, since to the force many gluon exchanges start to contribute. The scattering cross sections, which were up to the phase transition dominated by one gluon exchange, rise now to the value of a few $\mathrm{fm}^{2}$ and more, say $(50 \mathrm{fm})^{2}$. Although the colour phase transition is not yet well understood even for the first family quarks, the evaluation of what happens to the fifth family quarks and anti-quarks and coloured clusters of the fifth family quarks or anti-quarks can still be done as follows. At the interval, when the temperature $k_{b} T$ is considerably above the binding energy of the "dressed" first family quarks and anti-quarks into mesons or of the binding energy of the three first family quarks or anti-quarks into the first family baryons or antibaryons, which is $\approx$ a few $\mathrm{MeV}$ (one must be more careful with the mesons), the first family quarks and anti-quarks move in the plasma like being free. (Let us remind the reader that the nuclear interaction can be derived as the interaction among the clusters of quarks [19].) 25 years ago there were several proposals to treat nuclei as clusters of dressed quarks instead of as clusters of baryons. Although this idea was not very fruitful (since even models with nuclei as bound states of $\alpha$ particles work many a time reasonably) it also was not far from 
the reality. Accordingly it is meaningful to accept the description of plasma at temperatures above a few $10 \mathrm{MeV} / k_{b}$ as the plasma of less or more "dressed" quarks with the very large scattering amplitude $\left(\right.$ of $\left.\approx(50 \mathrm{fm})^{2}\right)$. The fifth family quarks and anti-quarks, heaving much higher mass (several ten thousands $\mathrm{GeV} / c^{2}$ to be compared with $\approx 300 \mathrm{MeV} / c^{2}$ ) than the first family quarks and accordingly much higher momentum, "see" the first family quarks as a "medium" in which they (the fifth family quarks) scatter among themselves. The fifth family quarks and anti-quarks, having much higher binding energy when forming a meson among themselves than when forming mesons with the first family quarks and anti-quarks (few thousand $\mathrm{GeV}$ to be compared with few $\mathrm{MeV}$ or few $10 \mathrm{MeV}$ ) and correspondingly very high annihilation probability and also pretty low velocities $\left(\approx 10^{-3} c\right)$, have during the scattering enough time to annihilate with their anti-particles. The ratio of the scattering time between two coloured quarks (of any kind) and the Hubble time is of the order of $\approx 10^{-18}$ and therefore although the number of the fifth family quarks and anti-quarks is of the order of $10^{-13}$ smaller than the number of the quarks and anti-quarks of the first family (as show the solutions of the Boltzmann equations presented in fig. III), the fifth family quarks and anti-quarks have in the first period of the colour phase transition (from $\approx \mathrm{GeV}$ to $\approx 10 \mathrm{MeV}$ ) enough opportunity to scatter often enough among themselves to deplete (their annihilation time is for several orders of magnitude smaller than the time needed to pass by). More detailed calculations, which are certainly needed, are under considerations. Let us still do rough estimation about the number of the coloured fifth family quarks (and anti-quarks). Using the expression for the thermally averaged cross section for scattering of a quark and an anti-quark and annihilating $\left(\langle\sigma v\rangle_{(q \bar{q})_{b}}\right.$ from Eq.(2) $)$ and correcting the part which determines the scattering cross section by replacing it with $\eta(50 \mathrm{fm})^{2} c$ (which takes into account the scattering in the plasma during the colour phase transition in the expanding universe) we obtain the expression $\left\langle\sigma v>_{(q \bar{q})_{b}}=\eta_{(q \bar{q})_{b}} \eta(50 \mathrm{fm})^{2} c \sqrt{\frac{E_{c_{5}}}{k_{b} T}} \ln \frac{E_{c_{5}}}{k_{b} T}\right.$, which is almost independent of the velocity of the fifth family quarks (which slow down when the temperature lowers). We shall assume that the temperature is lowering as it would be no phase transition and correct this fact with the parameter $\eta$, which could for a few orders of magnitude (say $10^{2}$ ) enlarge the depleting probability. Using this expression for $\langle\sigma v\rangle_{(q \bar{q})_{b}}$ in the expression for $\lambda=\frac{\langle\sigma v\rangle_{(q \bar{q})_{b}} m_{q_{5}} c^{2}}{h_{m}(\hbar c)^{3}}$, we obtain for a factor up to $10^{19}$ larger $\lambda$ than it was the one dictating the freeze out procedure of $q_{5}$ and $\bar{q}_{5}$ before the phase transition. Using then the equation $\frac{d Y_{q_{5}}}{d x}=\frac{\lambda_{c_{5}}}{x^{2}}\left(-Y_{q_{5}}^{2}\right)$ and integrating it from $Y_{1}$ which is the value from the fig. III at $1 \mathrm{GeV}$ up 
to the value when $k_{b} T \approx 20 \mathrm{MeV}$, when the first family quarks start to bindd into baryons, we obtain in the approximation that $\lambda$ is independent of $x$ (which is not really the case) that $\frac{1}{Y(20 \mathrm{MeV})}=10^{32} \frac{1}{2 \cdot 10^{5}}$ or $Y(20 \mathrm{MeV})=10^{-27}$ and correspondingly $n_{q_{5}}\left(T_{0}\right)=\eta^{-1} 10^{-24} \mathrm{~cm}^{-3}$. Some of these fifth family quarks can form the mesons or baryons and anti-baryons with the first family quarks $q_{1}$ when they start to form baryons and mesons. They would form the anomalous hydrogen in the ratio: $\frac{n_{a h}}{n_{h}} \approx \eta^{-1} \cdot 10^{-12}$, where $n_{a h}$ determines the number of the anomalous (heavy) hydrogen atoms and $n_{h}$ the number of the hydrogen atoms, with $\eta$ which might be bellow $10^{2}$. The best measurements in the context of such baryons with the masses of a few hundred $\mathrm{TeV} / \mathrm{c}^{2}$ which we were able to find were done 25 years ago [21]. The

authors declare that their measurements manifest that such a ratio should be $\frac{n_{a h}}{n_{h}}<10^{-14}$ for the mass interval between $10 \mathrm{TeV} / c^{2}$ to $10^{4} \mathrm{TeV} / c^{2}$. Our evaluation presented above is very rough and more careful treating the problem might easily lead to lower values than required. On the other side we can not say how trustable is the value for the above ratio for the masses of a few hundreds TeV. Our evaluations are very approximate and if $\eta=10^{2}$ we conclude that the evaluation agrees with measurements.

\section{DYNAMICS OF A HEAVY FAMILY BARYONS IN OUR GALAXY}

There are experiments [1, 2] which are trying to directly measure the dark matter clusters. Let us make a short introduction into these measurements, treating our fifth family clusters in particular. The density of the dark matter $\rho_{d m}$ in the Milky way can be evaluated from the measured rotation velocity of stars and gas in our galaxy, which appears to be approximately independent of the distance $r$ from the center of our galaxy. For our Sun this velocity is $v_{S} \approx(170-270) \mathrm{km} / \mathrm{s} . \rho_{d m}$ is approximately spherically symmetric distributed and proportional to $\frac{1}{r^{2}}$. Locally (at the position of our Sun) $\rho_{d m}$ is known within a factor of 10 to be $\rho_{0} \approx 0.3 \mathrm{GeV} /\left(c^{2} \mathrm{~cm}^{3}\right)$, we put $\rho_{d m}=\rho_{0} \varepsilon_{\rho}$, with $\frac{1}{3}<\varepsilon_{\rho}<3$. The local velocity distribution of the dark matter cluster $\vec{v}_{d m i}$, in the velocity class $i$ of clusters, can only be estimated, results depend strongly on the model. Let us illustrate this dependence. In a simple model that all the clusters at any radius $r$ from the center of our galaxy travel in all possible circles around the center so that the paths are spherically symmetrically distributed, the velocity of a cluster at the position of the Earth is equal to $v_{S}$, the velocity of our Sun in the absolute value, but has all possible orientations perpendicular to the radius $r$ with 
equal probability. In the model that the clusters only oscillate through the center of the galaxy, the velocities of the dark matter clusters at the Earth position have values from zero to the escape velocity, each one weighted so that all the contributions give $\rho_{d m}$. Many other possibilities are presented in the references cited in [1].

The velocity of the Earth around the center of the galaxy is equal to: $\vec{v}_{E}=\vec{v}_{S}+\vec{v}_{E S}$, with $v_{E S}=30 \mathrm{~km} / \mathrm{s}$ and $\frac{\vec{v}_{S} \cdot \vec{v}_{E S}}{v_{S} v_{E S}} \approx \cos \theta \sin \omega t, \theta=60^{\circ}$. Then the velocity with which the dark matter cluster of the $i$ - th velocity class hits the Earth is equal to: $\vec{v}_{d m E i}=\vec{v}_{d m i}-\vec{v}_{E}$. $\omega$ determines the rotation of our Earth around the Sun.

One finds for the flux of the dark matter clusters hitting the Earth: $\Phi_{d m}=\sum_{i} \frac{\rho_{d m i}}{m_{c_{5}}} \mid \vec{v}_{d m i}-$ $\vec{v}_{E} \mid$ to be approximately (as long as $\frac{v_{E S}}{\left|\vec{v}_{d m i}-\vec{v}_{S}\right|}$ is small) equal to

$$
\Phi_{d m} \approx \sum_{i} \frac{\rho_{d m i}}{m_{c_{5}}}\left\{\left|\vec{v}_{d m i}-\vec{v}_{S}\right|-\vec{v}_{E S} \cdot \frac{\vec{v}_{d m i}-\vec{v}_{S}}{\left|\vec{v}_{d m i}-\vec{v}_{S}\right|}\right\}
$$

Further terms are neglected. We shall approximately take that $\sum_{i}\left|\overrightarrow{v_{d m i}}-\overrightarrow{v_{S}}\right| \rho_{d m i} \approx$ $\varepsilon_{v_{d m S}} \varepsilon_{\rho} v_{S} \rho_{0}$, and correspondingly $\sum_{i} \vec{v}_{E S} \cdot \frac{\vec{v}_{d m i}-\vec{v}_{S}}{\left|\vec{v}_{d m i}-\vec{v}_{S}\right|} \approx v_{E S} \varepsilon_{v_{d m S}} \cos \theta \sin \omega t$, (determining the annual modulations observed by DAMA [1]). Here $\frac{1}{3}<\varepsilon_{v_{d m S}}<3$ and $\frac{1}{3}<\frac{\varepsilon_{v_{d m E S}}}{\varepsilon_{v_{d m S}}}<3$ are estimated with respect to experimental and (our) theoretical evaluations.

Let us evaluate the cross section for our heavy dark matter baryon to elastically (the excited states of nuclei, which we shall treat, I and Ge, are at $\approx 50 \mathrm{keV}$ or higher and are very narrow, while the average recoil energy of Iodine is expected to be $30 \mathrm{keV}$ ) scatter on an ordinary nucleus with $A$ nucleons $\sigma_{A}=\frac{1}{\pi \hbar^{2}}<\left|M_{c_{5} A}\right|>^{2} m_{A}^{2}$. For our heavy dark matter cluster is $m_{A}$ approximately the mass of the ordinary nucleus [23]. In the case of a coherent scattering (if recognizing that $\lambda=\frac{h}{p_{A}}$ is for a nucleus large enough to make scattering coherent when the mass of the cluster is $1 \mathrm{TeV}$ or more and its velocity $\approx v_{S}$ ), the cross section is almost independent of the recoil velocity of the nucleus. For the case that the "nuclear force" as manifesting in the cross section $\pi\left(r_{c_{5}}\right)^{2}$ in Eq.(1) brings the main contribution [24] the cross section is proportional to $(3 A)^{2}$ (due to the square of the matrix element) times $(A)^{2}$ (due to the mass of the nuclei $m_{A} \approx 3 A m_{q_{1}}$, with $m_{q_{1}} c^{2} \approx \frac{1 \mathrm{GeV}}{3}$ ). When $m_{q_{5}}$ is heavier than $10^{4} \mathrm{TeV} / c^{2}$ (Table I), the weak interaction dominates and $\sigma_{A}$ is proportional to $(A-Z)^{2} A^{2}$, since to $Z^{0}$ boson exchange only neutron gives an appreciable contribution. Accordingly we have, when the "nuclear force" dominates, $\sigma_{A} \approx \sigma_{0} A^{4} \varepsilon_{\sigma}$, with $\sigma_{0} \varepsilon_{\sigma}$, which is $\pi r_{c_{5}}^{2} \varepsilon_{\sigma_{n u c l}}$ and with $\frac{1}{30}<\varepsilon_{\sigma_{\text {nucl }}}<30$. $\varepsilon_{\sigma_{\text {nucl }}}$ takes into account the roughness with which we treat our heavy baryon's properties and the scattering procedure. 
When the weak interaction dominates, $\varepsilon_{\sigma}$ is smaller and we have $\sigma_{0} \varepsilon_{\sigma}=\left(\frac{m_{n_{1}} G_{F}}{\sqrt{2 \pi}} \frac{A-Z}{A}\right)^{2} \varepsilon_{\sigma_{\text {weak }}}$ $\left(=\left(10^{-6} \frac{A-Z}{A} \mathrm{fm}\right)^{2} \varepsilon_{\sigma_{\text {weak }}}\right), \frac{1}{10}<\varepsilon_{\sigma_{\text {weak }}}<1$. The weak force is pretty accurately evaluated, but the way how we are averaging is not.

\section{DIRECT MEASUREMENTS OF THE FIFTH FAMILY BARYONS AS DARK MATTER CONSTITUENTS}

We are making very rough estimations of what the DAMA [1] and CDMS [2] experiments are measuring, provided that the dark matter clusters are made out of our (any) heavy family quarks as discussed above. We are looking for limitations these two experiments might put on properties of our heavy family members. We discussed about our estimations and their relations to the measurements with R. Bernabei [14] and J. Filippini [14]. Both pointed out (R.B. in particular) that the two experiments can hardly be compared, and that our very approximate estimations may be right only within the orders of magnitude. We are completely aware of how rough our estimation is, yet we conclude that, since the number of measured events is proportional to $\left(m_{c_{5}}\right)^{-3}$ for masses $\approx 10^{4} \mathrm{TeV}$ or smaller (while for higher masses, when the weak interaction dominates, it is proportional to $\left.\left(m_{c_{5}}\right)^{-1}\right)$ that even such rough estimations may in the case of our heavy baryons say whether both experiments do at all measure our (any) heavy family clusters, if one experiment clearly sees the dark matter signals and the other does not (yet?) and we accordingly estimate the mass of our cluster.

Let $N_{A}$ be the number of nuclei of a type $A$ in the apparatus (of either DAMA [1, which has $4 \cdot 10^{24}$ nuclei per $\mathrm{kg}$ of $I$, with $A_{I}=127$, and $N a$, with $A_{N a}=23$ (we shall neglect $N a$ ), or of CDMS [2], which has $8.3 \cdot 10^{24}$ of $G e$ nuclei per $\mathrm{kg}$, with $A_{G e} \approx 73$ ). At velocities of a dark matter cluster $v_{d m E} \approx 200 \mathrm{~km} / \mathrm{s}$ are the $3 A$ scatterers strongly bound in the nucleus, so that the whole nucleus with $A$ nucleons elastically scatters on a heavy dark matter cluster. Then the number of events per second $\left(R_{A}\right)$ taking place in $N_{A}$ nuclei is due to the flux $\Phi_{d m}$ and the recognition that the cross section is at these energies almost independent of the velocity equal to

$$
R_{A}=N_{A} \frac{\rho_{0}}{m_{c_{5}}} \sigma(A) v_{S} \varepsilon_{v_{d m S}} \varepsilon_{\rho}\left(1+\frac{\varepsilon_{v_{d m E S}}}{\varepsilon_{v_{d m S}}} \frac{v_{E S}}{v_{S}} \cos \theta \sin \omega t\right)
$$


Let $\Delta R_{A}$ mean the amplitude of the annual modulation of $R_{A}$

$$
\Delta R_{A}=R_{A}\left(\omega t=\frac{\pi}{2}\right)-R_{A}(\omega t=0)=N_{A} R_{0} A^{4} \frac{\varepsilon_{v_{d m E S}}}{\varepsilon_{v_{d m S}}} \frac{v_{E S}}{v_{S}} \cos \theta
$$

where $R_{0}=\sigma_{0} \frac{\rho_{0}}{m_{c_{5}}} v_{S} \varepsilon, R_{0}$ is for the case that the "nuclear force" dominates $R_{0} \approx$ $\pi\left(\frac{3 \hbar c}{\alpha_{c} m_{q_{5}} c^{2}}\right)^{2} \frac{\rho_{0}}{m_{q_{5}}} v_{S} \varepsilon$, with $\varepsilon=\varepsilon_{\rho} \varepsilon_{v_{d m E S}} \varepsilon_{\sigma_{n u c l}}$. $R_{0}$ is therefore proportional to $m_{q_{5}}^{-3}$. We estimated $10^{-4}<\varepsilon<10$, which demonstrates both, the uncertainties in the knowledge about the dark matter dynamics in our galaxy and our approximate treating of the dark matter properties. (When for $m_{q_{5}} c^{2}>10^{4} \mathrm{TeV}$ the weak interaction determines the cross section $R_{0}$ is in this case proportional to $m_{q_{5}}^{-1}$.) We estimate that an experiment with $N_{A}$ scatterers should measure the amplitude $R_{A} \varepsilon_{c u t A}$, with $\varepsilon_{c u t ~}$ A determining the efficiency of a particular experiment to detect a dark matter cluster collision. For small enough $\frac{\varepsilon_{v_{d m E S}}}{\varepsilon_{v_{d m S}}} \frac{v_{E S}}{v_{S}} \cos \theta$ we have

$$
R_{A} \varepsilon_{\text {cut } A} \approx N_{A} R_{0} A^{4} \varepsilon_{\text {cut } A}=\Delta R_{A} \varepsilon_{c u t A} \frac{\varepsilon_{v_{d m S}}}{\varepsilon_{v_{d m E S}}} \frac{v_{S}}{v_{E S} \cos \theta} .
$$

If DAMA [1] is measuring our heavy family baryons scattering mostly on $I$ (we neglect the same number of $N a$, with $A=23$ ), then the average $R_{I}$ is

$$
R_{I} \varepsilon_{\text {cut dama }} \approx \Delta R_{d a m a} \frac{\varepsilon_{v_{d m S}}}{\varepsilon_{v_{d m E S}}} \frac{v_{S}}{v_{E S} \cos 60^{0}},
$$

with $\Delta R_{\text {dama }} \approx \Delta R_{I} \varepsilon_{\text {cut dama }}$, this is what we read from their papers [1]. In this rough estimation most of unknowns about the dark matter properties, except the local velocity of our Sun, the cut off procedure $\left(\varepsilon_{\text {cut dama }}\right)$ and $\frac{\varepsilon_{v_{d m S}}}{\varepsilon_{v_{d m E S}}}$, (estimated to be $\frac{1}{3}<\frac{\varepsilon_{v_{d m S}}}{\varepsilon_{v_{d m E S}}}<3$ ), are hidden in $\Delta R_{\text {dama }}$. If we assume that the Sun's velocity is $v_{S}=100,170,220,270 \mathrm{~km} / \mathrm{s}$, we find $\frac{v_{S}}{v_{E S} \cos \theta}=7,10,14,18$, respectively. (The recoil energy of the nucleus $A=I$ changes correspondingly with the square of $\left.v_{S}.\right)$ DAMA/NaI, DAMA/LIBRA [1] publishes $\Delta R_{d a m a}=$ 0.052 counts per day and per $\mathrm{kg}$ of $\mathrm{NaI}$. Correspondingly is $R_{I} \varepsilon_{\text {cut dama }}=0,052 \frac{\varepsilon_{v_{d m S}}}{\varepsilon_{v_{d m E S}}} \frac{v_{S}}{v_{S E} \cos \theta}$ counts per day and per kg. CDMS should then in 121 days with $1 \mathrm{~kg}$ of Ge $(A=73)$ detect $R_{\text {Ge }} \varepsilon_{\text {cut } c d m s} \approx \frac{8.3}{4.0}\left(\frac{73}{127}\right)^{4} \frac{\varepsilon_{\text {cut cdms }}}{\varepsilon_{\text {cut dama }}} \frac{\varepsilon_{v_{d m S} S}}{\varepsilon_{v_{d m E S}}} \frac{v_{S}}{v_{S E} \cos \theta} 0.052 \cdot 121$ events, which is for the above measured velocities equal to $(10,16,21,25) \frac{\varepsilon_{\text {cut } c d m s}}{\varepsilon_{\text {cut dama }}} \frac{\varepsilon_{v_{d m S}}}{\varepsilon_{v_{d m E S}}}$. CDMS [2] has found no event.

The approximations we made might cause that the expected numbers $(10,16,21,25)$ multiplied by $\frac{\varepsilon_{c u t} G e}{\varepsilon_{c u t} I} \frac{\varepsilon_{v_{d m S}}}{\varepsilon_{v_{d m E S}}}$ are too high (or too low!!) for a factor let us say 4 or 10 . If in the near future CDMS (or some other experiment) will measure the above predicted events, then there might be heavy family clusters which form the dark matter. In this case the DAMA experiment puts the limit on our heavy family masses (Eq. 12 ). 
Taking into account all the uncertainties mentioned above, with the uncertainty with the "nuclear force" cross section included (we evaluate these uncertainties to be $10^{-4}<$ $\varepsilon "<3 \cdot 10^{3}$ ), we can estimate the mass range of the fifth family quarks from the DAMA experiments: $\left(m_{q_{5}} c^{2}\right)^{3}=\frac{1}{\Delta R_{\text {dama }}} N_{I} A^{4} \pi\left(\frac{3 \hbar c}{\alpha_{c}}\right)^{2} \rho_{0} c^{2} v_{E S} \cos \theta \varepsilon^{\prime \prime}=\left(0.3 \cdot 10^{7}\right)^{3} \varepsilon^{\prime \prime}\left(\frac{0.1}{\alpha_{c}}\right)^{2} \mathrm{GeV}$. The lower mass limit, which follows from the DAMA experiment, is accordingly $m_{q_{5}} c^{2}>200$ TeV. Observing that for $m_{q_{5}} c^{2}>10^{4} \mathrm{TeV}$ the weak force starts to dominate, we estimate the upper limit $m_{q_{5}} c^{2}<10^{5} \mathrm{TeV}$. Then $200 \mathrm{TeV}<m_{q_{5}} c^{2}<10^{5} \mathrm{TeV}$.

Let us at the end evaluate the total number of our fifth family neutrons $\left(n_{5}\right)$ which in $\delta t=121$ days strike $1 \mathrm{~kg}$ of Ge and which CDMS experiment could detect, that is $R_{G e} \delta t \varepsilon_{c u t} t_{G e}=N_{G e} \sigma_{0} \frac{\rho_{0}}{m_{c_{5}}} v_{S} A_{G e}^{4} \varepsilon \varepsilon_{c u t+G e}$ (Eq. 12, with $N_{G e}=8.3 \cdot 10^{24} / \mathrm{kg}$, with the cross section from Table If, with $A_{G e}=73$ and $1 \mathrm{~kg}$ of Ge, while $10^{-5}<\varepsilon \varepsilon_{c u t}<5 \cdot 10$. The coefficient $\varepsilon \varepsilon_{c u t_{G e}}$ determines all the uncertainties: about the scattering amplitudes of the fifth family neutrons on the Ge nuclei (about the scattering amplitude of one $n_{5}$ on the first family quark, about the degree of coherence when scattering on the nuclei, about the local density of the dark matter, about the local velocity of the dark matter and about the efficiency of the experiment). Quite a part of these uncertainties were hidden in the number of events the DAMA/LIBRA experiments measure, when we compare both experiments. If we assume that the fifth family quark mass $\left(m_{q_{5}}\right)$ is several hundreds $\mathrm{TeV}$, as evaluated (as the upper bound (Eq. 7)) when considering the cosmological history of our fifth family neutrons, we get for the number of events the CDMS experiment should measure: $\varepsilon \varepsilon_{\text {cut }_{G e}} \cdot 10^{4}$. If we take $\varepsilon \varepsilon_{c u t} t_{G e}=10^{-5}$, the CDMS experiment should continue to measure 10 times as long as they did.

Let us see how many events CDMS should measure if the dark matter clusters would interact weakly with the Ge nuclei and if the weak interaction would determine also their freezing out procedure, that is if any kind of WIMP would form the dark matter. One easily sees from the Boltzmann equations for the freezing out procedure for $q_{5}$ that since the weak massless boson exchange is approximately hundred times weaker than the one gluon exchange which determines the freeze out procedure of the fifth family quarks, the mass of such an object should be hundred times smaller, which means a few TeV. Taking into account the expression for the weak interaction of such an object with Ge nuclei, which leads to $10^{-2}$ smaller cross section for scattering of one such weakly interacting particle on one proton (see derivations in the previous section), we end up with the number of events 
which the CDMS experiment should measure: $\varepsilon \varepsilon_{c u t} 5 \cdot 10^{3}$. Since the weak interaction with the matter is much better known that the ("fifth family nuclear force") interaction of the

colourless clusters of $q_{5}\left(n_{5}\right)$, the $\varepsilon$ is smaller. Let us say $\varepsilon$ is $5 \cdot 10^{-4}$. Accordingly, even in the case of weakly interacting dark matter particles the CDMS should continue to measure to see some events.

\section{CONCLUDING REMARKS}

We estimated in this paper the possibility that a new stable family, predicted by the approach unifying spin and charges [5, 6, 8, to have the same charges and the same couplings to the corresponding gauge fields as the known families, forms baryons which are the dark matter constituents. The approach (proposed by S.N.M.B.) is to our knowledge the only proposal in the literature so far which offers the mechanism for generating families, if we do not count those which in one or another way just assume more than three families. Not being able so far to derive from the approach precisely enough the fifth family masses and also not (yet) the baryon asymmetry, we assume that the neutron is the lightest fifth family baryon and that there is no baryon - anti-baryon asymmetry. We comment what changes if the asymmetry exists. We evaluated under these assumptions the properties of the fifth family members in the expanding universe, their clustering into the fifth family neutrons, the scattering of these neutrons on ordinary matter and find the limit on the properties of the stable fifth family quarks due to the cosmological observations and the direct experiments provided that these neutrons constitute the dark matter.

We use the simple hydrogen-like model to evaluate the properties of these heavy baryons and their interaction among themselves and with the ordinary nuclei. We take into account that for masses of the order of $1 \mathrm{TeV} / \mathrm{c}^{2}$ or larger the one gluon exchange determines the force among the constituents of the fifth family baryons. Studying the interaction of these baryons with the ordinary matter we find out that for massive enough fifth family quarks $\left(m_{q_{5}}>10^{4} \mathrm{TeV}\right)$ the weak interaction starts to dominate over the "nuclear interaction" which the fifth family neutron manifests. The non relativistic fifth family baryons interact among themselves with the weak force only.

We study the freeze out procedure of the fifth family quarks and anti-quarks and the formation of baryons and anti-baryons up to the temperature $k_{b} T=1 \mathrm{GeV}$, when the 
colour phase transition starts which to our estimations depletes almost all the fifth family quarks and anti-quarks while the colourless fifth family neutrons with very small scattering cross section decouples long before (at $k_{b} T=100 \mathrm{GeV}$ ).

The cosmological evolution suggests for the mass limits the range $10 \mathrm{TeV}<m_{q_{5}} c^{2}<$ a few $\cdot 10^{2} \mathrm{TeV}$ and for the scattering cross sections $10^{-8} \mathrm{fm}^{2}<\sigma_{c_{5}}<10^{-6} \mathrm{fm}^{2}$. The measured density of the dark matter does not put much limitation on the properties of heavy enough clusters.

The DAMA experiments [1] limit (provided that they measure our heavy fifth family clusters) the quark mass to: $200 \mathrm{TeV}<m_{q_{5}} c^{2}<10^{5} \mathrm{TeV}$. The estimated cross section for the dark matter cluster to (elastically, coherently and nonrelativisically) scatter on the (first family) nucleus is in this case determined on the lower mass limit by the "fifth family nuclear force" of the fifth family clusters $\left(\left(3 \cdot 10^{-5} A^{2} \mathrm{fm}\right)^{2}\right)$ and on the higher mass limit by the weak force $\left(\left(A(A-Z) 10^{-6} \mathrm{fm}\right)^{2}\right)$. Accordingly we conclude that if the DAMA experiments are measuring our fifth family neutrons, the mass of the fifth family quarks is a few hundred $\mathrm{TeV} / c^{2}$.

Taking into account all the uncertainties in connection with the dark matter clusters (the local density of the dark matter and its local velocity) including the scattering cross sections of our fifth family neutrons on the ordinary nuclei as well as the experimental errors, we do expect that CDMS will in a few years measure our fifth family baryons.

Let us point out that the stable fifth family neutrons are not the WIMPS, which would interact with the weak force only: the cosmological behaviour (the freezing out procedure) of these clusters are dictated by the colour force, while their interaction with the ordinary matter is determined by the "fifth family nuclear force" if they have masses smaller than $10^{4} \mathrm{TeV} / c^{2}$.

In the ref. [20] 25] the authors study the limits on a scattering cross section of a heavy dark matter cluster of particles and anti-particles (both of approximately the same amount) with the ordinary matter, estimating the energy flux produced by the annihilation of such pairs of clusters. They treat the conditions under which would the heat flow following from the annihilation of dark matter particles and anti-particles in the Earth core start to be noticeable. Using their limits we conclude that our fifth family baryons of the mass of a few hundreds $\mathrm{TeV} / c^{2}$ have for a factor more than 100 too small scattering amplitude with the ordinary matter to cause a measurable heat flux on the Earth's surface. On the other 
hand could the measurements [21] tell whether the fifth family members do deplete at the colour phase transition of our universe enough to be in agreement with them. Our very rough estimation show that the fifth family members are on the allowed limit, but they are too rough to be taken as a real limit.

Our estimations predict that, if the DAMA experiments observe the events due to our (any) heavy family members, (or any heavy enough family clusters with small enough cross section), the CDMS experiments [2] will in the near future observe a few events as well. If CDMS will not confirm the heavy family events, then we must conclude, trusting the DAMA experiments, that either our fifth family clusters have much higher cross section due to the possibility that $u_{5}$ is lighter than $d_{5}$ so that their velocity slows down when scattering on nuclei of the earth above the measuring apparatus bellow the threshold of the CDMS experiment (and that there must be in this case the fifth family quarks-anti-quarks asymmetry) [17]) while the DAMA experiment still observes them, or the fifth family clusters (any heavy stable family clusters) are not what forms the dark matter.

Let us comment again the question whether it is at all possible (due to electroweak experimental data) that there exist more than three up to now observed families, that is, whether the approach unifying spin and charges by predicting the fourth and the stable fifth family (with neutrinos included) contradict the observations. In the ref. [18] the properties of all the members of the fourth family were studied (for one particular choice of breaking the starting symmetry). The predicted fourth family neutrino mass is at around $100 \mathrm{GeV} / c^{2}$ or higher, therefore it does not due to the detailed analyses of the electroweak data done by the Russian group [15] contradict any experimental data. The stable fifth family neutrino has due to our calculations considerably higher mass. Accordingly none of these two neutrinos contradict the electroweak data. They also do not contradict the nucleosynthesis, since to the nucleosynthesis only the neutrinos with masses bellow the electron mass contribute. The fact that the fifth family baryons might form the dark matter does not contradict the measured (first family) baryon number and its ratio to the photon energy density as well, as long as the fifth family quarks are heavy enough $(>1 \mathrm{TeV})$. All the measurements, which connect the baryon and the photon energy density, relate to the moment(s) in the history of the universe, when the baryons (of the first family) where formed $\left(m_{1} c^{2} \approx k_{b} T=1 \mathrm{GeV}\right.$ and lower) and the electrons and nuclei were forming atoms $\left(k_{b} T \approx 1 \mathrm{eV}\right)$. The chargeless (with respect to the colour and electromagnetic charges, not with respect to the weak charge) 
clusters of the fifth family were formed long before (at $k_{b} T \approx E_{c_{5}}$ (Table II). They manifest after decoupling from the plasma (with their small number density and small cross section) (almost) only their gravitational interaction.

Let the reader recognize that the fifth family baryons are not the objects-WIMPSwhich would interact with only the weak interaction, since their decoupling from the rest of the plasma in the expanding universe is determined by the colour force and their interaction with the ordinary matter is determined with the fifth family "nuclear force" (the force among the fifth family nucleons, manifesting much smaller cross section than does the ordinary "nuclear force") as long as their mass is not higher than $10^{4} \mathrm{TeV}$, when the weak interaction starts to dominate as commented in section IV.

Let us conclude this paper with the recognition: If the approach unifying spin and charges is the right way beyond the standard model of the electroweak and colour interaction, then more than three families of quarks and leptons do exist, and the stable (with respect to the age of the universe) fifth family of quarks and leptons is the candidate to form the dark matter. The assumptions we made (i. The fifth family neutron is the lightest fifth family baryon, ii. There is no fifth family baryon asymmetry), could be derived from the approach unifying spins and charges and we are working on these problems. The fifth family baryon anti-baryon asymmetry does not very much change the conclusions of this paper as long as the fifth family quarks's mass is a few hundreds TeV or higher.

\section{APPENDIX I. THREE FIFTH FAMILY QUARKS' BOUND STATES}

We look for the ground state solution of the Hamilton equation $H|\psi\rangle=E_{c_{5}}|\psi\rangle$ for a cluster of three heavy quarks with

$$
H=\sum_{i=1}^{3} \frac{p_{i}^{2}}{2 m_{q_{5}}}-\frac{2}{3} \sum_{i<j=1}^{3} \frac{\hbar c \alpha_{c}}{\left|\vec{x}_{i}-\vec{x}_{j}\right|},
$$

in the center of mass motion

$$
\vec{x}=\vec{x}_{2}-\vec{x}_{1}, \quad \vec{y}=\vec{x}_{3}-\frac{\vec{x}_{1}+\vec{x}_{2}}{2}, \quad \vec{R}=\frac{\vec{x}_{1}+\vec{x}_{2}+\vec{x}_{3}}{3},
$$

assuming the anti-symmetric colour part $\left(|\psi\rangle_{c, \mathcal{A}}\right)$, symmetric spin and weak charge part $\left(|\psi\rangle_{w \operatorname{spin}, \mathcal{S}}\right)$ and symmetric space part $\left(|\psi\rangle_{\text {space, } \mathcal{S}}\right)$. For the space part we

take the hydrogen-like wave functions $\psi_{a}(\vec{x})=\frac{1}{\sqrt{\pi a^{3}}} e^{-|\vec{x}| / a}$ and $\psi_{b}(\vec{y})=\frac{1}{\sqrt{\pi b^{3}}} e^{-|\vec{y}| / b}$, 
allowing $a$ and $b$ to adapt variationally. Accordingly $\left\langle\vec{x}_{1}, \vec{x}_{2}, \vec{x}_{3} \mid \psi\right\rangle_{\text {space } \mathcal{S}}=$ $\mathcal{N}\left(\psi_{a}(\vec{x}) \psi_{b}(\vec{y})+\right.$ symmetric permutations $) . \quad$ It $\quad$ follows $\left\langle\vec{x}_{1}, \vec{x}_{2}, \vec{x}_{3} \mid \psi\right\rangle_{\text {space }} \mathcal{S}=$ $\left.\mathcal{N}\left(2 \psi_{a}(\vec{x}) \psi_{b}(\vec{y})+2 \psi_{a}\left(\vec{y}-\frac{\vec{x}}{2}\right) \psi_{b}\left(\frac{\vec{y}}{2}+\frac{3 \vec{x}}{4}\right)\right)+2 \psi_{a}\left(\vec{y}+\frac{\vec{x}}{2}\right) \psi_{b}\left(\frac{\vec{y}}{2}-\frac{3 \vec{x}}{4}\right)\right)$.

The Hamiltonian in the center of mass motion reads $H=\frac{p_{x}^{2}}{2\left(\frac{m_{q_{5}}}{2}\right)}+\frac{p_{y}^{2}}{2\left(\frac{2 m_{q_{5}}}{3}\right)}+\frac{p_{R}^{2}}{2 \cdot 3 m_{q_{5}}}-$ $\frac{2}{3} \hbar c \alpha_{c}\left(\frac{1}{x}+\frac{1}{\left|\vec{y}+\frac{\vec{x}}{2}\right|}+\frac{1}{\left|\vec{y}-\frac{\vec{x}}{2}\right|}\right)$. Varying the expectation value of the Hamiltonian with respect to $a$ and $b$ it follows: $\frac{a}{b}=1.03, \frac{a \alpha_{c} m_{q_{5}} c^{2}}{\hbar c}=1.6$.

Accordingly we get for the binding energy $E_{c_{5}}=0.66 m_{q_{5}} c^{2} \alpha_{c}^{2}$ and for the size of the cluster $\sqrt{\left\langle\left|\vec{x}_{2}-\vec{x}_{1}\right|^{2}\right\rangle}=2.5 \frac{\hbar c}{\alpha_{c} m_{q_{5} c^{2}}}$.

To estimate the mass difference between $u_{5}$ and $d_{5}$ for which $u_{5} d_{5} d_{5}$ is stable we treat the electromagnetic $\left(\alpha_{e l m}\right)$ and weak $\left(\alpha_{w}\right)$ interaction as a small correction to the above calculated binding energy: $H^{\prime}=\alpha_{\text {elmw }} \hbar c\left(\frac{1}{x}+\frac{1}{\left|\vec{y}+\frac{\vec{x}}{2}\right|}+\frac{1}{\left|\vec{y}-\frac{\vec{x}}{2}\right|}\right) . \quad \alpha_{e l m w}$ stays for electromagnetic and weak coupling constants. For $m_{q_{5}}=200 \mathrm{TeV}$ we take $\alpha_{e l m w}=\frac{1}{100}$, then $\left|m_{u_{5}}-m_{d_{5}}\right|<\frac{1}{3} E_{c_{5}} \frac{\left(\frac{3}{2} \alpha_{e l m w}\right)^{2}}{\alpha_{c}^{2}}=0.5 \cdot 10^{-4} m_{q_{5}} c^{2}$.

\section{Acknowledgments}

The authors would like to thank all the participants of the workshops entitled "What comes beyond the Standard models", taking place at Bled annually (usually) in July, starting in 1998, and in particular H. B. Nielsen, since all the open problems were there very openly discussed.

[1] R. Bernabei, P. Belli, F. Cappella, R. Cerulli, F. Montecchia, F. Nozzoli, A. Incicchitti, D. Prosperi, C.J. Dai, H.H. Kuang, J.M. Ma, Z.P. Ye, "Dark Matter particles in the galactic halo: results and implications from DAMA/NaI", Int. J. Mod. Phys. D13 (2004) 2127-2160, astro-ph/0501412, astro-ph/0804.2738v1.

[2] Z. Ahmed et al., A Search for WIMPs with the First Five-Tower Data from CDMS, pre-print, astro-ph/0802.3530.

[3] S. Dodelson, Modern Cosmology, Academic Press Elsevier 2003.

[4] C. Amsler et al., Phys. Lett. B 667, 1 (2008). 
[5] A. Borštnik Bračič, N.S. Mankoč Borštnik, "On the origin of families of fermions and their mass matrices", hep-ph/0512062, Phys. Rev. D 74 (2006) 073013-16.

[6] N.S. Mankoč Borštnik, "Spin connection as a superpartner of a vielbein" Phys. Lett. B 292 (1992) 25-29, "Unification of spin and charges", Int. J. Theor. Phys. 40 (2001) 315-337, "From the starting Lagrange density to the effective fields for spinors in the Approach unifying spin and charges" in the Proceedings. hep-ph/0711.4681, 94-113.

[7] N.S. Mankoč Borštnik, "Spinor and vector representations in four dimensional Grassmann space", J. Math. Phys. 34 (1993) 3731-3745, "Unification of spin and charegs in Grassmann space?", Mod. Phys. Lett. A 10 (1995) 587-595.

[8] G. Bregar, M. Breskvar, D. Lukman, N.S. Mankoč Borštnik, "Predictions for four families by the Approach unifying spin and charges", New J. of Phys. 10 (2008) 093002.

[9] N.S. Mankoč Borštnik, to be published in the Proceedings to Bled 2009 workshop, the talk can be found on www.cosmovia.org.

[10] N.S. Mankoč Borštnik, H.B. Nielsen, "How to generate spinor representations in any dimension in terms of projection operators", "How to generate families of spinor", J. of Math. Phys. 43 (2002), (5782-5803), J. of Math. Phys. 44 (2003) 4817-4827.

[11] G. Bregar, N.S. Mankoč Borštnik, arXiv:0812.0510 [hep-ph], 2-12, Proceedings to the $11^{\text {th }}$ workshop "What Comes Beyond the Standard Models", Bled July 15-25, 2008, DMFAzaložništvo, Jadranska 19, 1000 Ljubljana, Slovenija, no. 1724, 1-13.

[12] A. De Rujula, Phys. Rev. D 12 (1975) 147.

[13] G. Bregar, M. Khlopov, N.S. Mankoč Borštnik, work in progress.

[14] We thank cordialy R. Bernabei (in particular) and J. Filippini for very informative discussions by emails and in private communication.

[15] V.A. Novikov, L.B. Okun,A.N. Rozanov, M.I. Vysotsky, "Extra generations and discrepancies of electroweak precision data", Phys. Lett. B 529 (2002) 111, hep-ph/0111028, V.A. Novikov, A.N. Rozanov, M.I. Vysotsky, arXiv:0904.4570, private discussions.

[16] A. Shchukin, talk presented at $14^{\text {th }}$ Lomonosov Conference on Elementary Particle Physics, Moscow August 19/25, 2009.

[17] Maxim Yu. Khlopov, "Composite dark matter from stable charged constituents", pre-print, astro-ph/0806.3581.

[18] M. Breskvar, D. Lukman, N.S. Mankoč Borštnik, hep-ph/0612250, p.25-50. 
[19] N. Mankoč Borštnik, M. Cvetič-Krivec, B. Golli, M. Rosina, Proc. Int. Conf on Nuclear Structure, Amsterdam (1982), Physic Letters 93B, 489-491 (1980), J. Phys. G7, 1385-1393 (1981), Rosina, Phys. Lett. 99C, 486-488 (1981).

[20] G.D. Mack, J.F. Beacom , G. Bertone, Phys. Rev. D 76 (2007) 043523.

[21] M. Verkerk, G. Grynberg, B. Pichard, M. Spiro, S. Zylberajch, M.E. Goldberg, P. Fayet, "Search for superheavy hydrogen in sea watter, Phys. Rev. Lett. 8 (1992) 1116-1119.

[22] Let us tell that a simple bag model evaluation does not contradict such a simple Bohr-like model.

[23] Let us illustrate what is happening when a very heavy $\left(10^{4}\right.$ times or more heavier than the ordinary nucleon) cluster hits the nucleon. Having the "nuclear force" cross section of $10^{-8}$ $\mathrm{fm}^{2}$ or smaller, it "sees" with this cross section a particular quark, which starts to move. But since at this velocities the quark is tightly bound into a nucleon and nucleon into the nucleus, the hole nucleus is forced to move with the moving quark.

[24] The very heavy colourless cluster of three quarks, hitting with the relative velocity $\approx 200 \mathrm{~km} / \mathrm{s}$ the nucleus of the first family quarks, "sees" the (light) quark $q_{1}$ of the nucleus through the cross section $\pi\left(r_{c 5}\right)^{2}$. But since the quark $q_{1}$ is at these velocities strongly bound to the proton and the proton to the nucleus, the hole nucleus takes the momentum.

[25] The referee of PRL suggested that we should comment on the paper [20]. 